\title{
Management of an Annual Fishery in the Presence of Ecological Stress: The Case of Shrimp and Hypoxia
}

\author{
Ling Huang, ${ }^{*}$ Martin D. Smith ${ }^{\dagger}$ \\ Nicholas School of the Environment \\ Duke University
}

\begin{abstract}
The emergence of ecosystem-based management suggests that traditional fisheries management and protection of environmental quality are increasingly interrelated. Fishery managers, however, have limited control over most sources of marine and estuarine pollution and at best can only adapt to environmental conditions. We develop a bioeconomic model of optimal harvest of an annual species that is subject to an environmental disturbance. We parameterize the model to analyze the effect of hypoxia (low dissolved oxygen) on the optimal harvest path of brown shrimp, a commercially important species that is fished in hypoxic waters in the Gulf of Mexico and in estuaries in the southeastern United States. We find that hypoxia alters the qualitative pattern of optimal harvest and shifts the season opening earlier in the year; more severe hypoxia leads to even earlier season openings. Failure to adapt to hypoxia leads to greater losses when the effects of hypoxia are more severe. However, rent gains from adapting fishery management to hypoxia are relatively small compared to rent losses from the hypoxia effect itself. This suggests that it is critical for other regulatory agencies to control estuarine pollution, and fishery managers need to generate value from the fishery resources through other means such as rationalization.
\end{abstract}

Keywords: bioeconomics; hypoxia; adaptive policy; ecosystem-based management

JEL Classification Numbers: Q22 Q53.

*Post-Doctoral Fellow, Fisheries Center, the University of British Columbia. 1.huang@fisheries.ubc.ca .

${ }^{\dagger}$ Associate Professor of Environmental Economics, Nicholas School of the Environment and Department of Economics, Duke University, Box 90328, Durham, NC 27708, USA. Corresponding author. Email: marsmith@duke.edu t: 01-919-613-8028 f: 01-919-684-8741. 


\section{Introduction}

With the current shift towards ecosystem-based management, traditional natural resource management and protection of environmental quality are increasingly interrelated. Environmental quality affects the productivity of the natural resource base, and resource extraction can influence environmental quality. In spite of these feedbacks, natural resource extraction and environmental quality are typically managed by different agencies.

For fisheries resources, managers often have little or no control over environmental quality. Of particular importance for many fisheries is the degradation of estuarine habitat in which many fish spend critical life stages. Estuaries often experience hypoxia (low dissolved oxygen) and other environmental disturbances due to nutrient pollution from agricultural runoff and atmospheric deposition from fossil fuel combustion (NRC 2000). Hypoxia, in turn, can lead to juvenile mortality and a range of sub-lethal effects that have growth consequences. Several recent studies document the deleterious effects of hypoxia on fishery productivity by analyzing micro-level data from fishermen (Huang et al. 2010; Massey et al. 2006; Lipton and Hicks 2003). These studies quantify the increased value to the fishery from improved environmental quality, but they presume that a regulator would be able to influence pollution. Unfortunately, fishery managers cannot control the flows of nutrients into estuaries and can only react to pollution levels. In this paper, we explore how fishery managers can set optimal harvest policies that condition on fluctuating environmental conditions in estuaries.

Previous bioeconomic studies have examined related issues but have not considered how environmental conditions may influence harvest rates within a season. In a theoretical study, McConnell and Strand (1989) demonstrate that optimal management of the fishery is necessary to capture gains from water quality improvements. They compare only steady states under open access and optimal management. In a similar vein, Smith (2007) develops a bioeconomic model calibrated to the North Carolina blue crab fishery. He analyzes the full dynamic path of fishery rents and shows that gains from reduced hypoxia are higher when the fishery is rationalized. However, these gains are dwarfed by the gains from rationalization even with no water quality improvements. Most recently, Carson et al. (2009) theoretically model optimal harvest under cyclical population dynamics. They show that when ENSO cycles induce periodically fluctuating growth rates, optimal harvest is asynchronous with the ENSO cycle. Although they address related problems, these studies do not analyze within-season dynam- 
ics, which are likely to be especially important for annual fisheries such as shrimp and squid; the size structure and hence available biomass for harvest of an annual species change dramatically over the course of a year. In this paper, we examine specifically how the path of optimal management changes in the presence of hypoxia.

We analyze the brown shrimp fishery. Brown shrimp (Farfantepenaeus aztecus) is one of the largest fisheries in the U.S. with nominal ex vessel landings over the past decade ranging from over $\$ 156$ million to nearly $\$ 377$ million. ${ }^{1}$ Moreover, brown shrimp are fished in areas that experience seasonal hypoxia, most notably in the Gulf of Mexico. Like other shrimp species, brown shrimp have an annual life cycle and a weak stock recruitment relationship, i.e. recruitment is virtually uncorrelated with the previous years' stock. As a result of these reproductive features, shrimp are managed with seasonal closures rather than total allowable catches. Nonetheless, our results indicate that there are opportunities to design more efficient harvest policies by adjusting season closures to take environmental disturbances into consideration.

We develop a size-structured bioeconomic model that accounts for growth and aging of brown shrimp based on Clark (1990). We incorporate the effect of hypoxia directly into shrimp growth. In a numerical illustration, we calibrate the level of hypoxia to recent empiricallybased growth effects from hypoxia in the Neuse River in North Carolina (Huang et al. 2010). Our results show that fishery managers should open the shrimp season earlier in years with more severe hypoxia. In a pristine year, there is a larger payoff from waiting to open the season and allowing shrimp to grow larger. However, greater environmental stress increases the opportunity cost of waiting. Moreover, the benefits of adaptating fishery management to hypoxia are greater when the effects of hypoxia are more severe.

\section{The Model}

In this section, we develop a stylized bioeconomic model to consider the optimal harvest strategy of an annual species in the presence of an environmental disturbance. Our motivating example and numerical results are based on brown shrimp subjected to hypoxia, but our model could apply more broadly to other annual species like squid and other seasonally varying pollution problems. Brown shrimp have annual life cycles, usually spawning in spring with very

\footnotetext{
${ }^{1}$ See http:/ / www.st.nmfs.noaa.gov/st1/commercial/index.html.
} 
high fecundity rates and completing their life cycles in winter in deep offshore areas. However, they spend most of their lives growing in estuarine and inshore areas where many shrimp fisheries occur. The features of annual life cycles and extremely high fecundity rates imply that the annual shrimp stock recruitment could be regarded as being independent of the previous year's stock and harvest. As such, it is reasonable to assume exogenous recruitment. We derive the optimal policy from the perspective of a social planner and the planner's objective is to choose the harvest path to maximize the total present value profits from harvesting.

\subsection{The problem of optimal Harvest}

Let $p(t)$ indicate the price of fish per unit at time $\mathrm{t}$ and $Q(t)$ be the harvest in weight at time $\mathrm{t}$. Let $C$ denote the cost function depending on effort at time $t(E(t))$. Then the social planner's problem can be formally described as:

$$
\begin{gathered}
\max _{\{E(t)\}} \int_{0}^{T}(p(t) * Q(E(t), X(t))-C(E(t))) e^{-r t} d t \\
\text { s.t. } X(t)=f(X(t), t)-Q(t)
\end{gathered}
$$

This equation illustrates that the planner is to maximize the total present value profits by choosing the optimal effort path between 0 and $T$ (a maximum time after which individuals are dead or highly dispersed), where $r$ is the instantaneous discount rate. The constraint describes the stock $(X(t))$ dynamics consisting of a growth component $(f(X(t), t))$ and harvest $(Q(t))$. Combining biological models for annual species (Fontaine and Neal, 1971; McCoy, 1968; Minello et al. 1989) and bioeconomic models for the hypoxia effect (Huang et al. 2010), we can specify stock dynamics as the following:

$$
\dot{X(t)}=X(t)\left[-m(t)-\phi A(t)+\frac{\dot{W(t)}}{W(t)}\right]-Q(t)
$$

Unlike the canonical bioeconomic model of the fishery that gives rise to the Fundamental Equation of Renewable Resource Economics (Clark and Munro 1975; Conrad 1999), this state equation is non-autonomous and the stock dynamics are directly influenced by ecological stress. Appendix A provides a detailed description of how this equation is developed, but 
it is worth noting some details here. First, the natural mortality rate, $m(t)$, is time-dependent. This feature can account for the fact that, ceteris paribus, smaller fish have more predators than larger ones. Second, $W(t)$ is the fish weight per individual. Although our model describes a single cohort, we still must track how the cohort grows within the season. Third, $\phi A(t)$ is the instantaneous effect of environmental stress on stock dynamics. Like natural mortality, the effect of environmental stress is proportional to stock size and time-dependent. This specification allows for lethal effects such that the environmental stress increases natural mortality, but it also accounts for sub-lethal effects. For example, environmental stress such as hypoxia can reduce growth because animals expend energy avoiding low oxygen areas (Craig and Crowder 2005). A virtue of our specification is that one can use an empirical estimate of the total effect of stress on the population without having to distinguish the lethal and sub-lethal effects from observational data (something that has not been done in the literature on hypoxia).

Following Huang et al. (2010), $A(t)$ is a binary indictor of whether there is environmental stress ( 1 if stress exists at time $t$ and 0 otherwise). For our case study of hypoxia, $A(t)$ is coded 1 if bottom dissolved oxygen is below $2 \mathrm{mgl}^{-1}$ (the standard measure of hypoxia). The coefficient, $\phi$, is the marginal effect of environmental stress on the fish stock. Note that we measure the total stress effect by integrating stress events over time because they have a cumulative effect (See Equation 18 in Appendix A). The intuition for a cumulative effect is the same as having a dose-response relationship for a toxin or a carcinogen. Because the instantaneous effect is the product of this constant and the occurrence of stress, the instantaneous effect is time-dependent. In the analytical model and in our numerical simulations, we treat $A(t)$ as if it is known throughout the planning period. This is a simplification in order to gain analytical and numerical insights about the potential for managers to adapt to environmental stresses. In reality, managers would not know the exact timing of episodic environmental stresses like hypoxia ex ante, but they can often predict the seasonal concentration of stress events. Relaxing the assumption of a deterministic $A(t)$ could be a fruitful direction for future research.

Furthermore, we specify the catch and cost functions with standard assumptions from the literature. More specifically,

$$
Q(t)=q * E(t) * X(t)
$$


where $q$ is a constant, called the catchability coefficient. For the cost function, we assume that the total cost is proportional to the effort:

$$
C(t)=c * E(t)
$$

In this expression, $c$ is a positive constant. With all the expressions of the profit structure and stock dynamics, we can fully characterize the social planner's problem in the following:

$$
\begin{aligned}
\max _{\{E(t)\}} \int_{0}^{T}(p(t) q E(t) X(t)-c E(t)) e^{-r t} d t \\
\text { s.t. } \dot{X(t)}=X(t) *\left(-m(t)-\phi A(t)+\frac{\dot{W(t)}}{W(t)}\right)-q E(t) X(t) \\
m(t)=\beta(L(t))^{\rho} \\
W(t)=\omega L(t)^{\eta} \\
L(t)=L_{\infty}\left(1-e^{\delta t}\right) \\
0 \leq E(t) \leq \bar{E} \\
N_{0} \quad \text { known }
\end{aligned}
$$

Where $L(t)$ is the fish length, $L_{\infty}$ is the maximum length and $N_{0}$ is the initial stock number. The biological equations and parameters are introduced and defined in the Appendix A. $\bar{E}$ is the capacity limit for each day's effort; i.e. the effort level is never greater than this limit. Note that we use a finite-horizon model to characterize the annual fishery.

\subsection{The solution to the social planner's problem}

In this section, we derive the solution to the annual fishery in the presence of ecological stress. With the model specification in the social planner's problem (Equation 6), the solution is the admissible path $\{E(t), X(t)\}$ that maximizes the total present value profit, where $E(t)$ is the control variable and $X(t)$ is the state variable. As a practical matter, what comes out of the optimization as well are season opening and closing times, $T_{0}$ and $T_{1}$ respectively, but it is unnecessary to model them as choice variables.

We can use the current value Hamiltonian to derive the necessary conditions for the op- 
timal paths of stock and effort. Let $g(t)$ denote the expression for the non-autonomous stock dynamics $-m(t)-\phi A(t)+\frac{W(t)}{W(t)}$. The current value Hamiltonian is:

$$
\widetilde{H}=p(t) q E(t) X(t)-c E(t)+\lambda(t) X(t) *(g(t)-q E(t))
$$

The current-value co-state variable $\lambda(t)$ represents the shadow value of additional stock, i.e. the benefit of increasing the stock marginally. Note that the Hamiltonian is linear in the control. As a result, the first necessary condition is:

$$
\frac{\partial \widetilde{H}}{\partial E}=p(t) q X(t)-c-\lambda(t) * q X(t)=\sigma(t)
$$

where $\sigma(t)$ is the switching function as defined in Clark (1990). The problems in which the Hamiltonian is linear in the control are characterized by a bang-bang solution such that the control is at the maximum level, the minimum level, or follows a singular path (Spence and Starrett 1975; Clark 1990). For our problem, the bang-bang solution is:

$$
E(t)^{*}= \begin{cases}0 & \text { if } \sigma(t)<0 \\ E^{\text {singular }} & \text { if } \sigma(t)=0 \\ \bar{E} & \text { if } \sigma(t)>0\end{cases}
$$

where $\mathrm{a}^{\prime * \prime}$ indicates the optimal path. Although our problem is non-autonomous, the intuition is the same as in other bioeconomic models in which stock growth and catch are balanced to maximize total present value benefits. Analyzing Equations 8 and 9 together, if the co-state variable is too high, we are better off allowing the stock to grow by not harvesting (exerting no effort). If the co-state variable is too low, we are better off harvesting at the maximum rate.

In addition to Equation 8, a second necessary condition (by Pontryagin's Maximum Principle) is:

$$
\dot{\lambda(t)}-r \lambda(t)=-p(t) q E(t)-\lambda(t)[g(t)-q E(t)]
$$




$$
\lambda(T)=0
$$

We also require the following transversality condition:

Lastly, because our environmental stress variable $A(t)$ is not continuous, we require additional conditions. Specifically, following Caputo (2005), we require:

$$
\lambda^{*}\left(\tau^{-}\right)=\lambda^{*}\left(\tau^{+}\right)
$$

where $\tau$ is a switch point in $A(t)$. See Tomiyama (1985) Theorem 1, Remark 4 for a detailed treatment of this issue. Because $A(t)$ is piecewise continuous, i.e. it is defined at every point along a continuous interval, Equation 12 holds at every switch point. Intuitively, by integrating Equation 10, the jumps in $g(t)$ become kinks such that continuity holds. The optimal policy is the triplet $\left\{X^{*}(t), E^{*}(t), \lambda^{*}(t)\right\}$ that solves equations 3,8 , and 10 by implementing the control rule in Equation 9 with the requirement that equations 11 and 12 hold. Pragmatically, this amounts to solving a system of ordinary differential equations (ODEs) in a piecewise fashion throughout the planning horizon.

For our particular problem, the optimal solution is characterized by three intervals: $\left[0, T_{0}\right]$, $\left(T_{0}, T_{1}\right],\left(T_{1}, T\right)$. The season is closed until time $T_{0}$. Then the season opens and maximum effort is exerted from $T_{0}$ to $T_{1}$. Then the season closes and no more effort is exerted. Appendix B provides analytical details and a description of our numerical routines. In addition to solving the ODEs for each combination of parameters in Matlab, we also discretize the problem and solve for the optimal path using Matlab's constrained optimization routine (FMINCON). The latter is computationally intensive but produces the same results with only minor discrepancies from the discretization.

\section{Materials and numerical results}

In this section, we first use the known parameters for shrimp growth and real hypoxia data in the Neuse River of North Carolina to illustrate the optimal harvest path for brown shrimp. Then, using numerical simulations, we calculate the efficiency loss for the cases when the 
policy has not taken hypoxia into account. The parameters in Equations 14 to 16 in Appendix A for brown shrimp are available in the scientific literature. Table 1 lists the parameter values and sources we use for the simulations. Among these parameters, the constant exponential discount rate is set to be 0.0005 . Thus the daily discount rate is $0.05 \%$, and the annual discount rate is about $17 \%$.

The hypoxia data we use are collected by the USGS (The United States Geological Survey) for the Neuse River estuary. This area experiences moderate to severe and recurring hypoxia, mostly in the summer months, causing fish kills and other negative ecological damage (Paerl et al.1998, Lenihan et al. 2001, Eby et al. 2005). The USGS data record measurements of bottom and top dissolved oxygen and other water quality variables every 15 minutes from three moorings in the Neuse River. Since shrimp live near the bottom, we create the daily bottom dissolved oxygen values by taking an average of each day's bottom dissolved oxygen measurements from three moorings. However, these continuous dissolved oxygen values cannot be directly used since laboratory experiments show that a tolerable lower bound is a threshold for shrimp's normal growth. Specifically, Renaud (1986) shows that brown shrimp avoid areas with levels $<2 \mathrm{mgl}^{-1}$. Therefore, we use $2 \mathrm{mgl}^{-1}$ as a threshold to indicate whether it is a hypoxic day. Mathematically, if the daily dissolved oxygen measurement is $<2 \mathrm{mgl}^{-1}$, the hypoxia indicator $(A(t))$ is 1 , otherwise 0 . The average number of hypoxic days from 1999 to 2005 is 61 days per year. We use 2004 hypoxia data since it is the worst hypoxia year between 1999 and 2005 so that we can see the worst hypoxia effects in these years.

Before we analyze the hypoxia effect on the optimal harvest strategy, we first derive the optimal strategy when there is no hypoxia. Figure 3 shows the optimal harvest and stock paths with different parameter values. The optimal effort level is a constant over time and equal to the capacity limit $(\bar{E})$ for these parameter values. The three panel rows in Figure 3 describe three different scenarios with alternative catchability coefficients $(q)$, marginal cost coefficients $(c)$ and shrimp prices $(p)$, respectively. We assume a constant within-season price in our initial simulations as in Béné and Doyen (2000). The plain lines in all panels of Figure 3 depict the baseline case with $p=2, c=100, q=0.001$ and hypoxia effect $(\phi)$ equal to 0 .

The lines with diamonds in the first panel row of Figure 3 depict the cases with higher catchability. The left column of the first row describes the optimal harvest paths when the catchability coefficients are different, while the right column depicts the corresponding stock paths. We find that the baseline optimal harvest has an inverse U-shape with a peak in the 
summer time, which is consistent with the actual seasonal shrimp fishery. In these two cases, the starting point of harvest is similar, while a higher catchability leads to a higher peak harvest and a later season closing date. Intuitively, higher catchability implies that it is profitable to fish the stock to a lower level.

The second row of Figure 3 depicts the scenarios when $c$ varies. Specifically, the lines with diamonds describe the case when marginal cost, $c$, is higher. From this figure, we find that a higher marginal cost coefficient shortens the shrimp harvest season. It also suggests that the managers should delay the season opening date. Similarly, the last row of Figure 3 illustrates the case when the shrimp price is higher at $\$ 4$ per pound. This figure suggests that if shrimp prices increase, the optimal fishing season extends for a longer period. The changes in marginal cost and price have similar effects on shifting the optimal harvest and stock paths. The intuition is that with lower shrimp price and higher fishing cost, it is more profitable to wait longer for the stock to grow larger. In the extreme case, if the marginal cost is extremely high or the price is extremely low, the season is only opened for a short amount of time or the season is not opened at all. An extremely short (or instantaneous) season could also occur if we removed the capacity constraint. In this case, the problem becomes like an optimal slaughter problem in aquaculture (Bjørndal, 1988). That is, the harvest sector waits until the resource growth is balanced by the time value of money and then harvests the entire year's catch instantaneously. This extreme interpretation is contingent on the weak stock recruitment relationship.

Overall, the results in Figure 3 are consistent with other bioeconomic models of shrimp fisheries. Most studies of the Gulf of Mexico shrimp fisheries use mathematical programming models that have far more parameters and equations to represent the fishery than our optimal control framework. Nevertheless, they find that optimal harvest paths peak in the middle of the season and recommend seasonal closures as part of optimal management (Önal et al. 1991; Nance et al. 1994; Önal 1996).

With regards to the hypoxia effect, we can derive different optimal harvest and stock paths with different hypoxia coefficients. These paths are illustrated in Figure 4 . The hypoxia indexes are depicted in the top panel of this figure. Recall that if the dissolved oxygen is less than 2 $m g l^{-1}$, the hypoxia index is equal to 1 , otherwise 0 . From the figure, we see that most of the hypoxia days occur from May to August. The optimal harvest paths for three different hypoxia effects (i.e., no hypoxia effect, $\phi=0.003$ and $\phi=0.009$ ) are illustrated in the middle panel. As 
usual, the plain lines are the baseline scenario with $p=2, c=100, q=0.001$ and $\phi=0$. The lines with diamonds and the lines with triangles depict increasing hypoxia effects. Comparing these three cases, we find that a higher hypoxia effect makes the optimal harvest start and peak earlier. Correspondingly, the optimal stock level is lower with the earlier peak time (bottom panel). Thus with greater environmental stress, we need to start fishing earlier. The optimal harvest path is shifted to the left by the hypoxia because the pristine stock (stock without harvest) is shifted to the left by the hypoxia. Mathematically, the larger the shift in the pristine stock from greater hypoxia, the larger the shift in the optimal harvest path. More intuitively, hypoxia decreases the benefits of waiting to harvest.

From Figure 4, the optimal harvest paths suggest that we should set an earlier season opening date if there is hypoxia. In other words, ignoring hypoxia might lead to a suboptimal policy, which again will lead to efficiency loss. In the shrimp fishery of North Carolina, one of the current adopted policies is to set the season opening date. Moreover, Texas uses a seasonal closure policy to allow shrimp to grow larger. Thus, it is interesting to compare some policy scenarios when hypoxia is partially or totally ignored in fishery management.

Figures 5 depicts the situations in which we follow different harvest strategies. The difference between the top panel and the bottom panel is that they illustrate the cases of $\phi=0.003$ (less severe hypoxia effect) and $\phi=0.009$ (more severe hypoxia effect), respectively. The parameters other than $\phi$ are all the same as the baseline scenario. The plain lines depict the optimal harvest paths that take hypoxia into account. If the social planner ignores the hypoxia effect, partially or totally, he could have two suboptimal polices. First, he only ignores the hypoxia in the beginning and sets the season opening date according to the no hypoxia case. After the suboptimal season opening, he realizes that hypoxia is a problem and adapts to it. It is too late to open the season early, but otherwise the regulator can follow the optimal strategy in the presence of hypoxia. We call this an "adaptive strategy". This result is depicted by the line with triangles, which shows that the new adaptive strategy under the suboptimal season opening policy is a little higher than the optimal one. Though formally the model is still deterministic in this case, the adaptive strategy is a plausible reaction of the regulator to a stochastic environmental stressor. That is, having observed hypoxia, the regulator adjusts the harvest rate and season closing time.

Beyond the adaptive strategy, a second alternative occurs if the social planner totally ignores the hypoxia and treats the problem as if there is no hypoxia. In this case, he will naively 
follow the baseline optimal effort path including opening and closing times. We call this a "non-adaptive strategy". So the season opening date is the same with the case of "adaptive strategy", and we use a dashed vertical line to show the same opening dates. The "nonadaptive strategy" is shown by the lines with diamonds in the figure.

In order to show clearly the magnitude of the efficiency loss due to suboptimal policies, we calculate the rents for different harvest strategies and report them in Table 2. This table describes sixteen combinations of parameters. While the line with "Optimal $(\phi=0)$ " describes the baseline case where there is no hypoixa, the line with "Optimal $(\phi>0)$ " reports the rent when there is hypoxia, the planner takes the hypoxia into account and derives the correct optimal strategy. The following lines denoted with "Adaptive strategy" and "Non-adaptive strategy "record results in two suboptimal policy cases described above. In this table, we calculate the hypoxia effect by dividing the rent under each cases by the baseline optimal rent with $\phi=0$ (no impact of hypoxia on the fishery). Policy loss is the difference between the adaptive strategy (or "Non-adaptive strategy") and the optimal rent with $\phi>0$. In addition, the policy loss percentage is derived by dividing the policy loss by the rent of "Optimal $(\phi>$ $0)^{\prime \prime}$. Given all these parameter combinations, the effort level is always binding and equal to $\bar{E}$. Table 2 shows that the rent loss due to hypoxia is big in both absolute and relative magnitude. For example, when $\phi=0.003, p=2, c=100$ and $q=0.001$, the loss due to hypoxia is about $29.52 \%$ of the baseline optimal rent. If the hypoxic coefficient, $\phi$, increases to 0.009 , the loss increases to $54.82 \%$. The policy loss is mostly low relative to the economic loss of hypoxia. With the same parameter combinations, if we use the adaptive harvest strategy, the loss would reduce to only $0.77 \%$ of the potential maximum rent. If we use the non-adaptive strategy, we lose $5.62 \%$ of the maximum rent with hypoxia. For case 3 with a higher hypoxia effect, the rent for the non-adaptive strategy is negative. This outcome occurs because the non-adaptive strategy naively follows the fishing opening and closing dates for the case where there is no hypoxia. In fact, for many fishing dates, the profit is negative, which results in negative total profits for some of the simulations. We also note that the policy losses for cases 5 and 6 with lower hypoxia effects are the same. This outcome is due to $T_{1}$ equaling to $T$ (the terminal time in our numerical simulations) so that the opening and closing dates are all the same. Because effort binds and the season remains open throughout the planning period, both strategies produce the same profits.

For the non-adaptive strategy, since there are negative fishing profits on some days and the 
model is deterministic, the regulator could easily avoid these losses in reality. When negative profit days are discarded from the non-adaptive strategy, the non-adaptive strategy is very close to the adaptive strategy and even coincides with it in some cases as discussed above. As such, we focus on the policy loss from the adaptive strategy. In all the sixteen cases in Table 2 , the biggest policy loss relative to hypoxia loss for adaptive strategy is $13.55 \%$. The fishery management gains from adapting to hypoxia are thus relatively small with these parameters.

\section{Discussion}

In this paper, we develop a stylized bioeconomic model for optimal harvest of an annual species (shrimp) in the presence of environmental degradation (hypoxia) that has growth consequences. In the absence of hypoxia, the optimal harvest path is longer or shorter depending on price and cost. Because of exogenous recruitment and the annual nature of the fishery, the problem is analogous to more controlled systems like rotationally managed forests or aquaculture ponds (Bjørndal 1988; Guttormsen 2008). If one eliminates the capacity constraint, the optimal strategy for wild shrimp harvest would be to wait for the shrimp to grow and harvest all at once, balancing shrimp and price growth over time with the cumulative effects of natural mortality and discounting.

When hypoxia is introduced, we find that the optimal harvest path changes qualitatively. In particular, as hypoxia worsens, the optimal season opening shifts earlier. The intuition follows from a standard capital-theoretic view of natural resources. Hypoxia slows the internal rate of return of the fish stock and thus shifts more harvest toward the present due to discounting. Conversely, in a pristine year managers are better off waiting longer to open the season. Under hypoxia, the path of optimal harvest also becomes adaptive. However, quantitatively the consequences are rather small. Previous empirical work finds that hypoxia in North Carolina's Neuse River has sizable effects on shrimp harvest- in the range of 13\% (Huang et al. 2010) - and our bioeconomic model finds comparable rent losses from hypoxia under the optimal policy for some parameter combinations. Yet, when managers fail to adapt to hypoxia and instead implement what would be optimal under no hypoxia, the rent losses are small. That is, the difference between following the optimal policy under hypoxia and following a naive policy with respect to the season opening is small. An important caveat is that our hypoxia scenarios are based on North Carolina's Neuse River and not the more sizable and notorious dead 
zone in the Gulf of Mexico. The benefits of adaptation to hypoxia could be more substantial in this much larger fishery that is subject to more severe hypoxia. Indeed, our results show that more severe impacts of hypoxia increase the policy losses from following either sub-optimal strategy (adaptive or non-adaptive).

Our quantitative findings constitute a negative result of sorts; we set out to find how fishery managers can adapt to water pollution that they are unable to control and generate more value from fishery resources, but we find only small potential changes in aggregate outcomes. Nonetheless, our results suggest important policy implications. First, because fishery managers are extremely limited in their ability to adapt to water pollution, reducing pollution takes on greater importance for other regulatory agencies. Second, fishery managers must find ways to generate value through some other means. One possibility is generating value through rationalization. For instance, Smith (2007) finds that the benefits from rationalization for North Carolina blue crabs are more than an order of magnitude larger than the benefits from reducing nutrient pollution and the resulting hypoxia. Similarly, the gains from some form of rationalization of the shrimp fishery will likely outweigh gains from reduced nutrient pollution and almost certainly would be larger than gains from optimal adaptation to hypoxia. Many caveats exist in our analysis. For example, all the prices in the numerical simulations are assumed constant over time as in Béné and Doyen (2000). However, in practice shrimp prices fluctuate during the season due in part to changing size structure within the season and the premium for larger shrimp. The bang-bang solution indicates that fluctuations of prices can switch the effort on and off over time, which complicates the problem. In addition, the capacity limit parameter potentially has normative implications. With a high enough capacity limit, a fishery that would most likely be highly overcapitalized, the solution moves toward an optimal slaughter problem in which all harvest is taken instantaneously. The temporal adaptation to hypoxia could become more important in this case. With a lower capacity limit, in contrast, we stretch the season out longer, and it may be less important to open the season early as a hypoxia adaptation strategy because the season already begins early in the fish's life history. Lastly, uncertainty about stock, catchability, and the cost function could change the nature of this problem, which we leave to future research.

Although our motivating example in this paper is hypoxia, the recent Deepwater Horizon Oil Spill presented fishery managers with an interesting parallel. The spill occurred before the shrimp season. Fishery managers decided to declare emergency early season openings to 
375 allow shrimp fishermen to catch some shrimp before stocks were inundated with oil from the 376 spill. Our model provides a theoretical justification for this action. 


\section{Acknowledgments}

378 The authors thank three anonymous referees for helpful comments. Financial support for this project was provided by the National Oceanic and Atmospheric administration (NOAA) Center for Sponsored Coastal Ocean Research under award \# NA05NOS4781197 to J.K. Craig, L.B. Crowder, M.D. Smith (Duke University). The views expressed herein are those of the authors and do not necessarily reflect the view of NOAA or any of its sub-agencies. 


\section{References}

[1] Béné, C., Doyen L., 2000. Storage and Viability of a Fishery with Resource and Market Dephased Seasonalities. Environmental and Resource Economics 15, 1-26.

[2] Bjørndal, T., 1988. Optimal harvesting of farmed fish. Marine Resource Economics 5(2), 13959.

[3] Caputo, M.R., 2005. Foundations of Dynamic Economic Analysis: Optimal Control Theory and Applications. New York: Cambridge University Press.

[4] Carson, R., Granger, C., Jackson, J., Schlenker, W., 2008. Fisheries management under cyclical population dynamics. Environmental and Resource Economics 42(3), 379-410.

[5] Clark, C. W., 1990. Mathematical bioeconomics: The optimal management of renewable resources (2nd edition). Wiley-Intersciences, New-York.

[6] Clark, C.W., Munro. G.R., 1975. The economics of fishing and modern capital theory: a simplified approach. Journal of Environmental Economics and Management 2, 92-106.

[7] Conrad, J.M., 1999. Resource Economics. Cambridge: Cambridge University Press.

[8] Craig, J.K., Crowder, L.B., 2005. Hypoxia-induced habitat shifts and energetic consequences in Atlantic croaker and brown shrimp on the Gulf of Mexico shelf. Marine Ecology Progress Series 294: 79-94.

[9] Eby, L.A., Crowder, L.B., McClellan, C.M., Peterson, C.H., Powers, M.J., 2005. Habitat degradation from intermittent hypoxia: impacts on demersal fishes. Marine Ecology Progress Series 291, 249-262.

[10] Fontaine, C.T., Neal, R.A., 1971. Length-weight relations for three commercially important penaeid shrimp in the Gulf of Mexico. Transactions of the American Fisheries Society 100, 584586.

[11] Guttormsen, A.G., 2008. Faustmann in the sea: optimal rotation in aquaculture. Marine Resource Economics 23(4), 401-10.

[12] Huang, L., Smith, M. D., Craig, J.K., 2010. Quantifying the Economic Effects of Hypoxia on a Fishery for Brown Shrimp Farfantepenaeus aztecus. Marine and Coastal Fisheries: Dynamics, Management, and Ecosystem Science 2:232-248. 
[13] Lenihan, H.S., Peterson, C.H., Byers, J.E., Grabowski, J.H., Thayer, G.W., Colby, D.R., 2001. Cascading of habitat degradation: oyster reefs invaded by refugee fishes escaping stress. Ecological Applications 11, 764-782.

[14] Lipton, D. W., Hicks, R., 2003. The cost of stress: Low dissolved oxygen and recreational striped bass (Morone saxatilis) fishing in the Patuxent River. Estuaries 26, 310-315.

[15] Massey, D.M., Newbold, S.C., Gentner, B., 2006. Valuing water quality changes using a bioeconomic model of a coastal recreational fishery. Journal of Environmental Economics and Management 52, 482-500.

[16] McCoy, E.G., 1968. Migration, growth and mortality of North Carolina pink and brown penaeid shrimps. North Carolina Department of Conservation and Development, Div. Commer. Sports Fish., Spec. Sci. Rep. 15. 26 pp.

[17] McConnell, K.E., Strand, I.E., 1989. Benefits from commercial fisheries when demand and supply depend on water quality. Journal of Environmental Economics and Management 17(3), 284-292

[18] Minello, T. J., Zimmerman, R. J., Martinez, E. X., 1989. Mortality of young brown shrimp Penaeus aztecus in estuarine nurseries. Transactions of the American Fisheries Society 118, 693708.

[19] Nance, J.M., Martinez, E.X., Klima, E.F., 1994. Feasibility of Improving the Economic Return from the Gulf of Mexico Brown Shrimp Fishery. North American Journal of Fisheries Management 14, 522-536.

[20] National Research Council (NRC), 2000. Clean Coastal Waters: Understanding and Reducing the Effects of Nutrient Pollution. National Academy Press, Washington, D.C.

[21] NCDENR-DMF, 2006. North Carolina Draft Shrimp Fishery Management Plan. Report, Apr.

[22] Önal, H., 1996. Optimum Management of a Hierarchically Exploited Open Access Resource: A Multilevel Optimization Approach. American Journal of Agricultural Economics 78, 448-459. 
[23] Önal H., McCarl, B.A., Griffin, W.L., Matlock, G., Clark, J., 1991. A Bioeconomic Analysis of the Texas Shrimp Fishery and Its Optimal Management. American Journal of Agricultural Economics 73, 1161-1170.

[24] Paerl, H.W., Pinckney, J.L., Fear J.M., Peierls, B.L., 1998. Ecosystem responses to internal watershed organic matter loading: Consequences for hypoxia and fish kills in the eutrophying Neuse River Estuary, North Carolina, USA. Marine Ecology Progress Series 166, 17-25.

[25] Renaud, M.L., 1986. Detecting and avoiding oxygen deficient sea water by brown shrimp, Penaeus aztecus (Ives), and white shrimp Penaeus setzferus(Linnaeus). Journal of Experimental Marine Biology and Ecology 98, 283-292.

[26] Smith, M.D., 2007. Generating Value in Habitat-dependent Fisheries: The Importance of Fishery Management Institutions. Land Economics 83, 59-73.

[27] Smith, M.D., Zhang, J., Coleman, F.C., 2008. Econometric Modeling of Fisheries with Complex Life Histories: Avoiding Biological Management Failures. Journal of Environmental Economics and Management 55, 265-280.

[28] Spence, M., Starrett, D., 1975. Most rapid approach paths in accumulation problems. International Economic Review 16: 388-403.

[29] Tahvonen, O., 2009. Economics of harvesting age-structured fish populations. Journal of Environmental Economics and Management 58, 281-299.

[30] Tomiyama, K.,1985. Two-stage optimal control problems and optimality conditions. Journal of Economic Dynamics and Control 9:317-337. 


\section{Appendix A:}

Our model is a size-structured single cohort model such that the state equation is a nonautonomous ordinary differential equation. We can decompose the growth component $(f(X(t), t))$ into biological growth rates that are available in the biology literature. Clark (1990) describes a similar model structure, and examples of recent work in fisheries bioeconomics using age- or size-structured models include Massey et al. (2006), Smith et al. (2008), and Tahvonen (2009). In general, the stock at time $t$ can be represented as the following:

$$
X(t)=N(t) * W(t)
$$

In this function, $N(t)$ is the number of individuals at time $t$ and $W(t)$ is the weight of each individual, so the total biomass $(X(t))$ is the product of number and weight. Furthermore, the number of fish can be modeled as a function of initial number and the mortality rate:

$$
N(t)=N_{0} e^{\int_{0}^{t}-m(u) d u}
$$

Here, $N_{0}$ is the initial number in each season, which is independent of last season's stock and determined by nature. That is, we assume exogenous recruitment at $\mathrm{t}=0$. In addition, $m(t)$ is the instaneous mortality rate, thus the total mortality at time $t$ is an integration of $m(t)$ over time. Because larger fish are typically subject to less predation, $m(t)$ can be further modeled as:

$$
m(t)=\beta(L(t))^{\rho}
$$

In this equation, $L(t)$ is the fish length and $\beta$ and $\rho$ are two parameters. We know that the number of fish decreases over time, so $\beta>0$, and $\rho<0$ reflects the marginal mortality rate decreasing over time since $L(t)$ is always positive. Jaw size of potential predators is the standard justification for the latter assumption. $L(t)$ itself can be modeled with a von-Bertalanffy 
function:

$$
L(t)=L_{\infty}\left(1-e^{\delta t}\right)
$$

In this von-Bertalanffy equation, $L_{\infty}$ is the maximum length of an individual fish. The parameter $\delta$ is less than zero to ensure that $L(t)$ increases over time. Finally, we decompose the number of fish $(N(t))$ to a function of $\mathrm{t}$. Recall that Equation 13 has two components, number of fish and weight $W(t)$, which can also be modeled with an allometric function of fish length with parameters $\omega$ and $\eta:$ :

$$
W(t)=\omega L(t)^{\eta}
$$

$$
X(t)=N(t) * W(t) * e^{\int_{0}^{t}-\phi A(u) d u}
$$

In this equation, $A(t)$ is an indicator of whether there is ecological stress, specifically, hypoxia in our case. The coefficient, $\phi$, is the marginal effect of stress on fish growth. Note that we measure the effect at time $t$ by integrating the effects over time because water quality problems like hypoxia can have cumulative effects (Huang et al. 2010).

With all the above equations, we are able to take the derivative of $X(t)$ with respect to $t$, 


$$
\begin{aligned}
\dot{X(t)} & =\frac{d(N(t) * W(t))}{d t} \\
& =\frac{d\left(N_{0} e^{\int_{0}^{t}(-m(u)-\phi A(u)) d u} * W(t)\right)}{d t} \\
& =N_{0} e^{\int_{0}^{t}(-m(u)-\phi A(u)) d u}(-m(t)-\phi A(t)) * W(t) *+N_{0} e^{\int_{0}^{t}(-m(u)-\phi A(u)) d u} * W(t) \\
& =N(t) W(t)\left[-m(t)-\phi A(t)+\frac{\dot{W(t)}}{W(t)}\right] \\
& =X(t)\left[-m(t)-\phi A(t)+\frac{\dot{W(t)}}{W(t)}\right]
\end{aligned}
$$

At this point, the stock is expressed as a differential equation of mortality rate, weight and the hypoxia effect without harvest. If we include harvest, this equation becomes:

$$
\dot{X(t)}=X(t)\left[-m(t)-\phi A(t)+\frac{\dot{W(t)}}{W(t)}\right]-Q(t)
$$

This equation is exactly the one we use as Equation 3 in the text for the stock dynamics. The shape of the pristine stock dynamics is depicted in Figure 1:

Figure 1: Biomass within the season

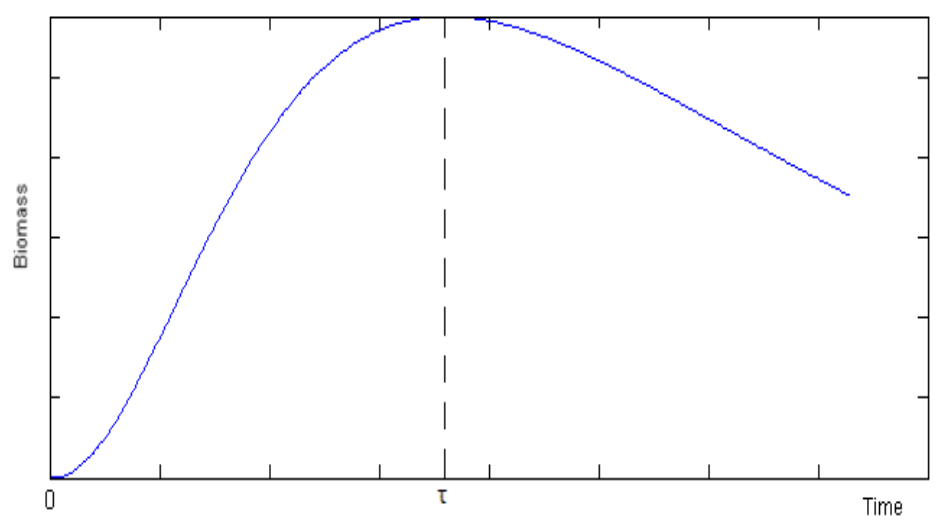

Note: Depicts the time path of a shrimp biomass index when there is no harvest and no hypoxic effects. The path is based on the population parameters in Table 1. 


\section{Appendix B:}

In this Appendix, we detail the numerical solution to the social planner's problem. Analytically, we can solve all of the ODEs such that only constants of integration are left to be determined. To begin, we integrate the state equation (Equation 3) to obtain:

$$
X(t)^{*}=\operatorname{cons}_{X} * W(t) * e^{\int_{0}^{t}-m(u)-\phi A(u)-q E(u) d u}
$$

where cons $_{X}$ is an unknown constant. This constant can be determined using the known initial condition $N_{0}$ (the number of individuals that recruit into the fishery in a given year). Thus, according to $X_{0}=N_{0} * W(0)$, we can solve for cons $_{X}$ to obtain:

$$
X(t)^{*}=N_{0} * W(t) * e^{\int_{0}^{t}-m(u)-\phi A(u)-q E(u) d u}
$$

To understand the evolution of the co-state variable, it is convenient to rewrite Equation 10 as:

$$
\dot{\lambda(t)}=q E(t)[\lambda(t)-p(t)]+\lambda(t)[r-g(t)]
$$

We now consider the extreme cases of when the control is at its maximum (Case A) and when the control is 0 (Case B). To understand the control rule, we can combine Equations 8 and 9. When the control is at the maximum (Case A), the following is true:

$$
\lambda(t)<p(t)-\frac{c}{q X(t)}
$$


Note that because $c, q$, and $X(t)$ are always positive, $\lambda(t)<p(t)$ in this case. And when the control is 0 (Case B), the following is true:

$$
\lambda(t)>p(t)-\frac{c}{q X(t)}
$$

For Case A, the sign of the first term in square brackets in Equation 22 is always negative as mentioned above. The sign of the second term in square brackets depends on whether growth, natural mortality, and the effects of ecological stress are larger or smaller than the discount rate. For our particular application of shrimp and hypoxia, $r-g(t)$ will be negative early in the season because shrimp are growing fast but positive late in the season once growth slows down and losses from natural mortality and hypoxia dominate. Figure 1 illustrates how growth slows and is overtaken by natural mortality even in the absence of hypoxia. If we do not consider the transversality condition, $\lambda(t)$ appears to decrease early in the season and increase late in the season. However, we need to account for the transversality condition. It turns out that transversality causes the rate of decrease of $\lambda(t)$ to slow late in the season, but $\lambda \dot{(t)}$ does not become positive.

Case B is more straightforward. The term in the first set of square brackets in Equation 22 drops out because the control is 0 . Thus, $\lambda(t)$ is decreasing early in the season. If not considering transversality condition, $\lambda(t)$ will increase late in the season. However, we need to take account of the transversality condition.

To satisfy the transversality condition, first solve Equation 22 to obtain:

$$
\lambda(t)=\frac{k-\int_{0}^{t} p(s) q E(s) e^{\int_{0}^{s}(-r+g(u)-q E(u)) d u} d s}{e^{\int_{0}^{t}(-r+g(u)-q E(u)) d u}}
$$

in which $k$ is an unknown constant. According to this equation, $\lambda(T)=0$ can only hold if $\lambda\left(T_{1}\right)=0$ because $\lambda(t)$ can be expressed as $\lambda\left(T_{1}\right) * \frac{e^{\int_{0}^{T_{1}}(-r+g(u)-q E(u)) d u}}{e^{\int_{0}^{t}(-r+g(u)-q E(u)) d u}}$ if $t \geqslant T_{1}$. Thus, $\lambda(t)$ is equal to 0 if $t \geqslant T_{1}$. Putting these cases together with the transversality condition satisfied allows us to rule out a singular path for our application. At $t=0$, the opportunity cost of harvest is too high because the fish have not yet started to grow. Thus, we are in Case B (Equation 24). As the stock grows, increased $p(t)-\frac{c}{q X(t)}$ and decreased $\lambda(t)$ eventually hit the threshold level where $\sigma(t)=0$ that justifies the singular control. At this point, call it $T_{0}$, the 
control turns on. However, by Equation 22, turning on the control will reinforce the decrease in $\lambda(t)$. Consequently, the effort will switch to the maximum control (Case A). At the same time, $r-g(t)$ will grow and cut the decreasing speed of $\lambda(t)$ until $\lambda(t)=0$. As the season progresses, $X(t)$ becomes lower which decreases $p(t)-\frac{c}{q X(t)}$, eventually the threshold is reached again and the control passes through the singular control and goes straight to 0 . Call this point $T_{1}$. $\lambda(t)$ becomes zero afterwards and the co-state permanently remains above the threshold and the control never turns on again.

The following algorithm is used to determine the remaining constants of integration and the switch points in the control problem.

1) Conjecture a $T_{0}$;

2) Solve the state equation assuming no effort between 0 and $T_{0}$ to find $X\left(T_{0}\right)$;

3) At $T_{0}$, use the fact that $\lambda(t)=p(t)-\frac{c}{q X(t)}$ to obtain $\lambda\left(T_{0}\right)$;

4) Substitute $\lambda\left(T_{0}\right)$ into the solved co-state equation (Equation 25) and solve for the constant $k$;

5) Now simulate the state and co-state equations assuming effort is $\bar{E}$ and find when $\lambda$ crosses the threshold. Call that "possible $T_{1}$ ";

6) Simulate the co-state and state equations with $E=0$ from "possible $T_{1}$ " and check that the threshold is not crossed again.

7) Choose the $T_{0}$ that leads to the highest present value profits.

As a check on the above, we solve the problem without using optimal control theory and instead using Matlab's FMINCON and a discretization of the time and control space. Specifically, we discretize $E(t)$ from 0 to $\bar{E}, T_{0}$ from 0 to $T$, where $T$ is 365 days, and $T_{1}$ from $T_{0}$ to $T$ in each simulation. Then for each combination we calculate the total present value profits for every possible effort path. Lastly, we choose the highest profit level. The solutions are the same for all combinations of parameters that we report in Table 2; we find only slight differences that are attributable to the discretization.

Figure 2 exemplifies the dynamics of $\lambda(t)$ and $p(t)-\frac{c}{q X(t)}$ in a case. $T_{0}$ and $T_{1}$ are determined where these two lines meet. 
Figure 2: The Harvest Switch

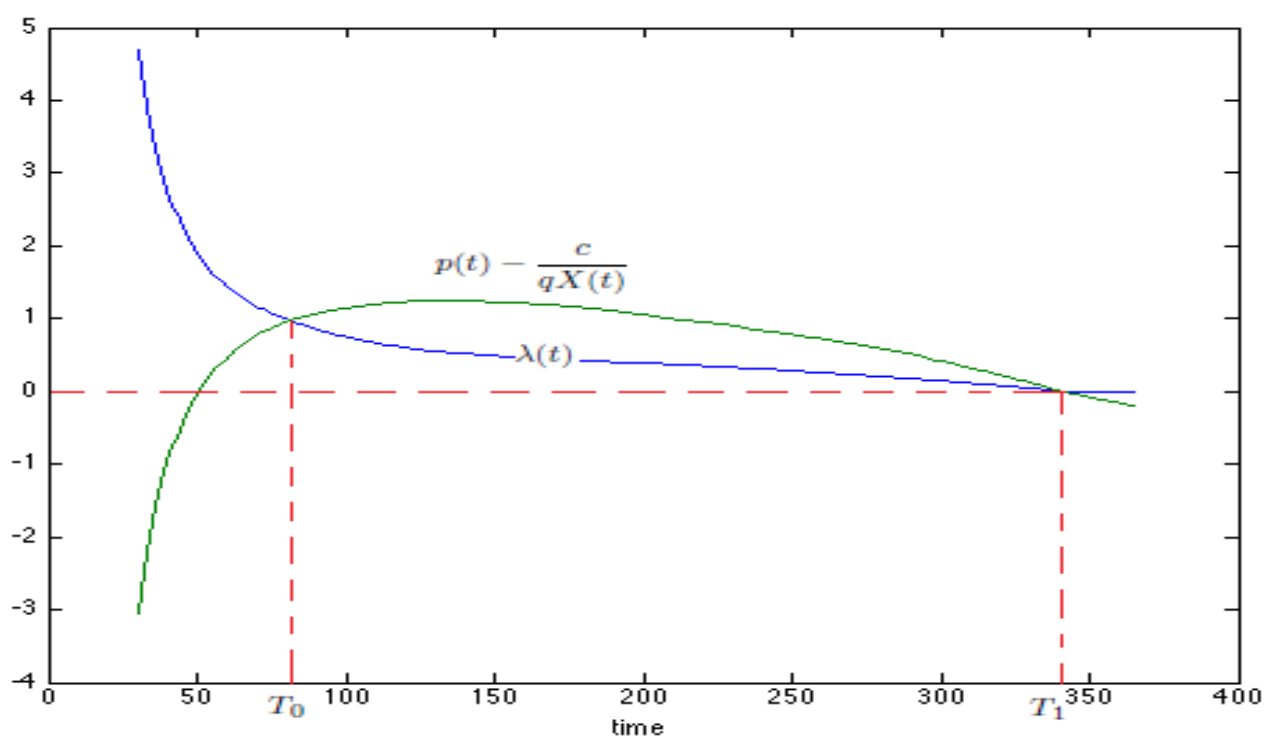

Note: Depicts the paths of $\lambda$ and $p(t)-\frac{c}{q X(t)}$. The paths are based on the population parameters in Table 1 and $p=2, c=150, q=0.001$ and $\phi=0.003$. 
Table 1: Parameter sources

\begin{tabular}{c|c|l|l}
\hline \hline Parameter & Value & Note & Parameter source \\
\hline$L_{\infty}$ & 177.7 & Von-Bertalanffy growth function & McCoy 1968 \\
$\delta$ & -0.0104 & Von-Bertalanffy growth function & McCoy 1968 \\
$\beta$ & 1.4866 & Natural mortality rate & Minello et al. 1989 \\
$\rho$ & -1.1163 & Natural mortality rate & Minello et al. 1989 \\
$\omega$ & $10.52^{*} \mathrm{E}-06$ & Allometric function(weight) & Fontaine and Neal 1971 \\
$\eta$ & 2.94 & Allometric function(weight) & Fontaine and Neal 1971 \\
$N_{0}$ & 100000 & Initial number of shrimp & \\
$r$ & 0.0005 & discount rate & \\
$c$ & 100 & fixed cost of fishing & \\
$p$ & 2 & price of per pound shrimp & \\
$q$ & 0.001 & catchability coefficient & \\
$\bar{E}$ & 2 & capacity limit & \\
\hline
\end{tabular}


Table 2: Efficiency loss due to hypoxia and suboptimal policies (US\$)

\begin{tabular}{|c|c|c|c|c|c|c|c|c|}
\hline & \multicolumn{4}{|c|}{$\phi=0.003$} & \multicolumn{4}{|c|}{$\phi=0.009$} \\
\hline & Rent & $\begin{array}{c}\text { Hypoxia } \\
\text { effect }\end{array}$ & $\begin{array}{l}\text { Policy loss } \\
\text { percentage }\end{array}$ & $\frac{\text { policy loss }}{\text { hypoxia loss }}$ & Rent & $\begin{array}{c}\text { Hypoxia } \\
\text { effect }\end{array}$ & $\begin{array}{l}\text { Policy loss } \\
\text { percentage }\end{array}$ & $\frac{\text { policy loss }}{\text { hypoxia loss }}$ \\
\hline \multicolumn{9}{|c|}{ Case 1: $\mathrm{p}=2, \mathrm{c}=100, \mathrm{q}=0.001$} \\
\hline Optimal $(\phi=0)$ & 27734 & & & & 27734 & & & \\
\hline Optimal $(\phi>0)$ & 19546 & $29.52 \%$ & & & 12531 & $54.82 \%$ & & \\
\hline Adaptive strategy & 19395 & $30.07 \%$ & $0.77 \%$ & $1.84 \%$ & 11742 & $57.66 \%$ & $6.30 \%$ & $5.19 \%$ \\
\hline Non-adaptive strategy & 18448 & $33.48 \%$ & $5.62 \%$ & $13.41 \%$ & 4774 & $82.79 \%$ & $61.90 \%$ & $51.02 \%$ \\
\hline \multicolumn{9}{|c|}{ Case $2: \mathrm{p}=2, \mathrm{c}=100, \mathrm{q}=0.002$} \\
\hline Optimal $(\phi=0)$ & 73189 & & & & 73189 & & & \\
\hline Optimal $(\phi>0)$ & 58875 & $19.56 \%$ & & & 42685 & $41.68 \%$ & & \\
\hline Adaptive strategy & 58350 & $20.27 \%$ & $0.89 \%$ & $3.67 \%$ & 39907 & $45.47 \%$ & $6.51 \%$ & $9.11 \%$ \\
\hline Non-adaptive strategy & 57673 & $21.20 \%$ & $2.04 \%$ & $8.40 \%$ & 34797 & $52.46 \%$ & $18.48 \%$ & $25.86 \%$ \\
\hline \multicolumn{9}{|c|}{ Case 3: $p=2, c=150, q=0.001$} \\
\hline Optimal $(\phi=0)$ & 10911 & & & & 10911 & & & \\
\hline Optimal $(\phi>0)$ & 6277 & $42.47 \%$ & & & 3640 & $66.64 \%$ & & \\
\hline Adaptive strategy & 5992 & $45.08 \%$ & $4.54 \%$ & $6.15 \%$ & 2853 & $73.85 \%$ & $21.62 \%$ & $10.82 \%$ \\
\hline Non-adaptive strategy & 4453 & $59.19 \%$ & $29.06 \%$ & $39.36 \%$ & -5363 & $149.15 \%$ & $247.34 \%$ & $123.82 \%$ \\
\hline \multicolumn{9}{|c|}{ Case $4: p=2, c=150, q=0.002$} \\
\hline Optimal $(\phi=0)$ & 52499 & & & & 52499 & & & \\
\hline Optimal $(\phi>0)$ & 40395 & $23.06 \%$ & & & 29102 & $44.57 \%$ & & \\
\hline Adaptive strategy & 39762 & $24.26 \%$ & $1.57 \%$ & $5.23 \%$ & 25932 & $50.60 \%$ & $10.89 \%$ & $13.55 \%$ \\
\hline Non-adaptive strategy & 38753 & $26.18 \%$ & $4.06 \%$ & $13.57 \%$ & 18229 & $65.28 \%$ & $37.36 \%$ & $46.47 \%$ \\
\hline \multicolumn{9}{|c|}{ Case 5: $\mathrm{p}=4, \mathrm{c}=100, \mathrm{q}=0.001$} \\
\hline Optimal $(\phi=0)$ & 103740 & & & & 103740 & & & \\
\hline Optimal $(\phi>0)$ & 83952 & $19.07 \%$ & & & 56955 & $45.10 \%$ & & \\
\hline Adaptive strategy & 83858 & $19.17 \%$ & $0.11 \%$ & $0.48 \%$ & 56193 & $45.83 \%$ & $1.34 \%$ & $1.63 \%$ \\
\hline Non-adaptive strategy & 83858 & $19.17 \%$ & $0.11 \%$ & $0.48 \%$ & 54815 & $47.16 \%$ & $3.76 \%$ & $4.57 \%$ \\
\hline \multicolumn{9}{|c|}{ Case 6: $\mathrm{p}=4, \mathrm{c}=100, \mathrm{q}=0.002$} \\
\hline Optimal $(\phi=0)$ & 194560 & & & & 194560 & & & \\
\hline Optimal $(\phi>0)$ & 165530 & $14.92 \%$ & & & 124780 & $35.87 \%$ & & \\
\hline Adaptive strategy & 165080 & $15.15 \%$ & $0.27 \%$ & $1.55 \%$ & 121890 & $37.35 \%$ & $2.32 \%$ & $4.14 \%$ \\
\hline Non-adaptive strategy & 165080 & $15.15 \%$ & $0.27 \%$ & $1.55 \%$ & 121620 & $37.49 \%$ & $2.53 \%$ & $4.53 \%$ \\
\hline \multicolumn{9}{|c|}{ Case $7: \mathrm{p}=4, \mathrm{c}=150, \mathrm{q}=0.001$} \\
\hline Optimal $(\phi=0)$ & 78516 & & & & 78516 & & & \\
\hline Optimal $(\phi>0)$ & 59031 & $24.82 \%$ & & & 38326 & $51.19 \%$ & & \\
\hline Adaptive strategy & 58842 & $25.06 \%$ & $0.32 \%$ & $0.97 \%$ & 37001 & $52.87 \%$ & $3.46 \%$ & $3.30 \%$ \\
\hline Non-adaptive strategy & 58151 & $25.94 \%$ & $1.49 \%$ & $4.52 \%$ & 28402 & $63.83 \%$ & $25.89 \%$ & $24.69 \%$ \\
\hline \multicolumn{9}{|c|}{ Case 8: $p=4, c=150, q=0.002$} \\
\hline Optimal $(\phi=0)$ & 170130 & & & & 170130 & & & \\
\hline Optimal $(\phi>0)$ & 140390 & $17.48 \%$ & & & 103170 & $39.36 \%$ & & \\
\hline Adaptive strategy & 139820 & $17.82 \%$ & $0.41 \%$ & $1.92 \%$ & 99100 & $41.75 \%$ & $3.94 \%$ & $6.08 \%$ \\
\hline Non-adaptive strategy & 139810 & $17.82 \%$ & $0.41 \%$ & $1.95 \%$ & 95123 & $44.09 \%$ & $7.80 \%$ & $12.02 \%$ \\
\hline
\end{tabular}

Note: Reports efficiency losses for different harvest strategies and parameter combinations, including low and high impacts of hypoxia $(\phi)$, two levels of catchability $(q)$, marginal harvest cost $(c)$, and shrimp price $(p)$. 
Figure 3: Optimal harvest without hypoxia
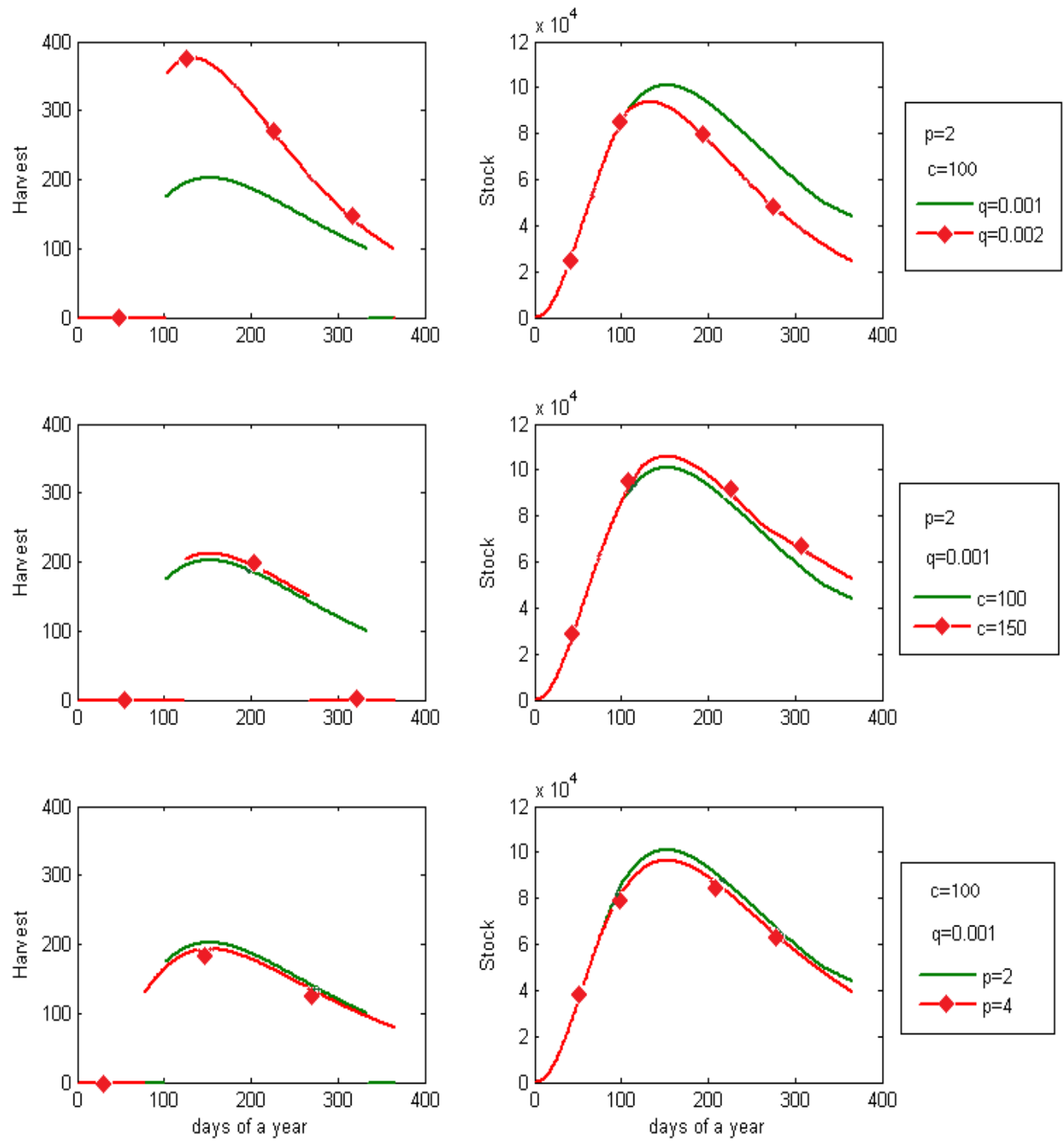

Note: Depicts the optimal harvest and corresponding stock paths when the catchability $(q)$, marginal harvest cost $(c)$ and shrimp price $(p)$ vary. 
Figure 4: Hypoxia effects
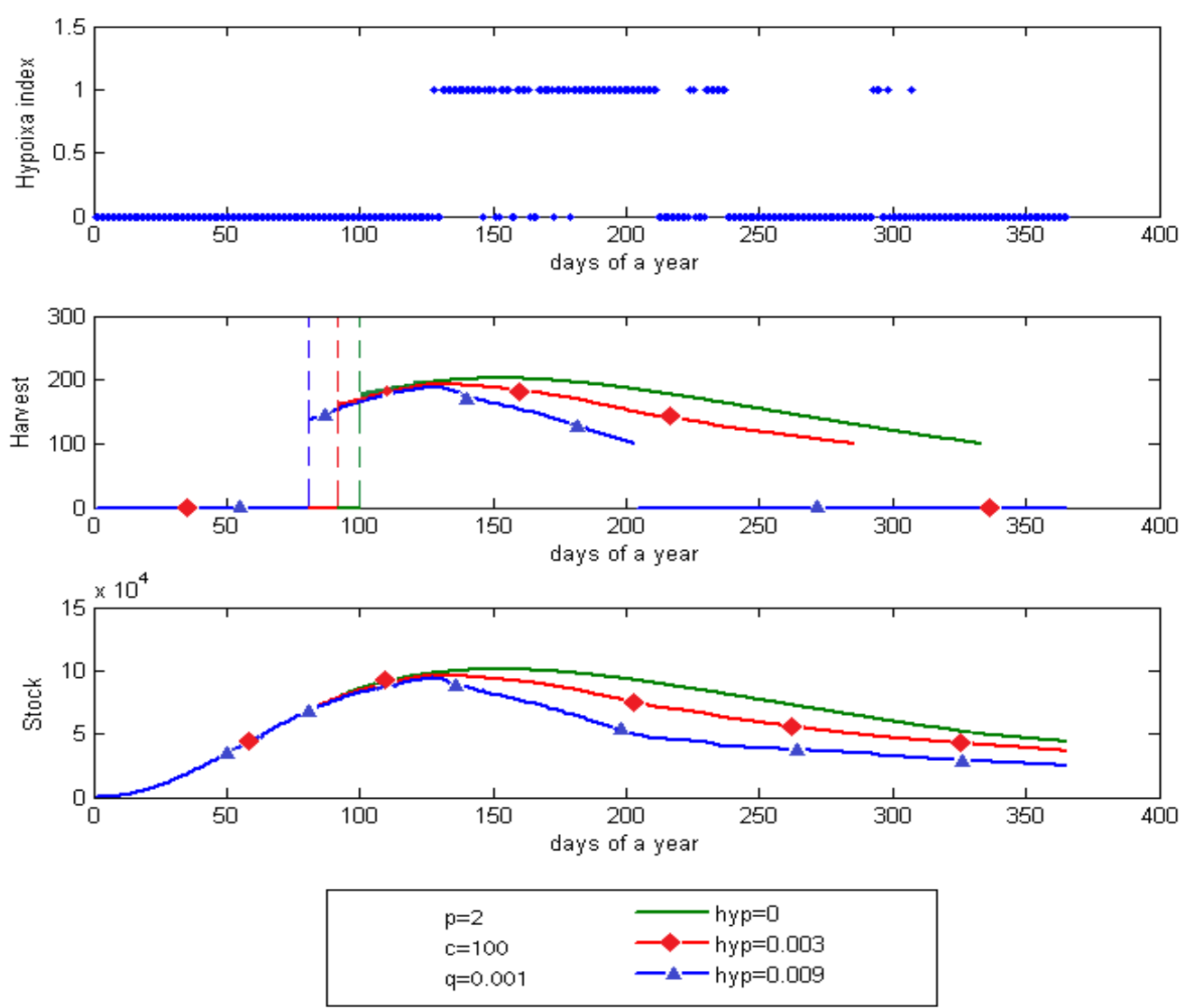

Note: Depicts hypoxia indexes (top panel), optimal harvest paths (middle panel) and stock paths (bottom panel) at different levels of hypoxia $(\phi)$. 
Figure 5: Harvest strategies under suboptimal policies
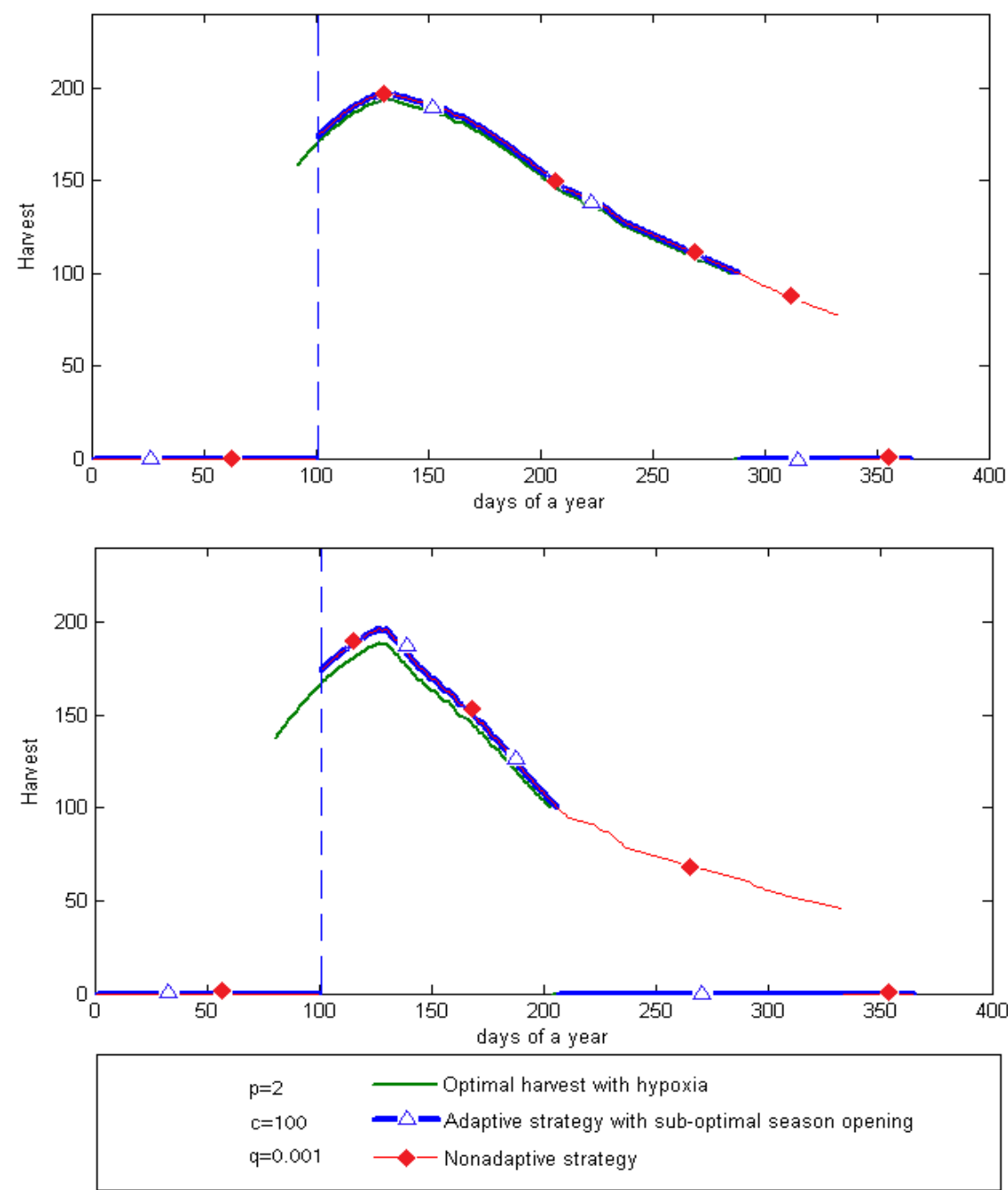

Note: Depicts four different harvest strategies for moderate impacts of hypoxia (top panel) and severe impacts of hypoxia (bottom panel). 